\title{
有限水库的最优控制一一折扣费用情形
}

\author{
林禁 \\ (香港中文大学统计系)
}

\section{\$1. 引市}

设有一个最大库容为 $V$ 的水库, 满足如下的条件:

(i) 记水库到时刻 $t$ 为止的进水量为 $X(t)$, 则 $\{X(t): t \geqslant 0\}$ 是一个具有非负漂移 $\mu$ 和方差参数 $\sigma^{2}$ 的 Wiener 过程.

(ii) 只有两个可能的放水率, 零和 $M$, 如果水库没有放水, 则它在时刻 $t$ 的库容 $Z(t)$ 是 一个漂移为 $\mu$ 方差为 $\sigma^{2}$ 的 Wiener 过程. 如果水库的放水率变为 $M$, 则 $Z(\varepsilon)$ 的漂移就变为 $\boldsymbol{\mu}-M$.

(iii) 初始库容为零, 并且在零和 $V$ 两处, $Z(t)$ 各有一个反射壁.

（iv）任何时刻，一旦付出开闸费用 $K_{1} M$, 水库的放水率就可以立即从 0 增为 $M$, 若付出 关闸费用, 水库的放水率就可以马上从 $M$ 减为 0 . 而且, 每放出一个单位的水, 其报酬为 $A$ （不失一般性, 可设 $A=1$ ).

(v) 赔款费用是按赔款率 $g(Z(t))$ 连续累积来计算的, 这里 8 是一个已知的连续函数. 例如, 8 可能是

$$
g(x)= \begin{cases}p M(a-x), & 0 \leqslant x \leqslant a, \\ 0, & a<x \leqslant v,\end{cases}
$$

其中 $p, a$ 是两个常数而 $x$ 是水库的库容.

我们将在下述一类策略中来研究水库的控制问题: $P_{\lambda, \mathrm{M}}^{M}$ 表示一个策略, 它的放水率保持 为一直到水库库容增加到 $\lambda(0<\lambda \leqslant V)$, 这时放水率从零增为 $M$, 然后保持这个放水率 $M$ 直到库容减少为 $\tau(0 \leqslant \tau<\lambda)$, 这时放水率才从 $M$ 减为番.

林埜 ${ }^{[1]}$ 通过解一个和费用泛函有关的微分方程的方法, 得到了长期平均费用的明显表达 式, 因此最优策略可以用数值方法来确定, 从而推广了 Faddy ${ }^{[2]}$, Zuckerman ${ }^{[3]}$ 还有 Attia 和 Brockwell ${ }^{43}$ 的研究成果.

本文乃是文献 [1] 的继续. 在 $\$ 2$, 我们先明显地确定了期望折扣总费用, 从而有可能用 数值分析的方法去求出其最优策略. 然后, 在 $\$ 3$, 我们建立了长期平均费用与期望折扣总 用间的关系。

\section{2. 期望折扣总费用函数}

有如在作者的论文 ${ }^{[1]}$ 中一样, 记 $Z(t)$ 为水库的库容, $Y(t)$ 为放水率 $(0$ 或 $M)$, 我们考 虑二维 Markov 过程 $W=(Z, Y)$, 其状态空间为

$$
s=([0, \lambda] \times\{0\}) \cup([\tau, V] \times\{M\}) \text {. }
$$

由条件 (iii), 起初 $W(0)=(0,0)$.

本文1984年12月 4 日收到. 
同样，我们定义如下一列的首次到达时间:

$$
\begin{aligned}
& T_{0}=\inf \{t \geqslant 0: Z(t)=\lambda\}, \\
& T_{0}^{\prime}=\inf \left\{t \geqslant T_{0}: Z(t)=\tau\right\},
\end{aligned}
$$

一般地

$$
\begin{aligned}
& T_{n}=\inf \left\{t \geqslant T_{n-1}^{\prime}: Z(t)-\lambda\right\}, n=1,2 \cdots, \\
& T_{n}^{\prime}=\inf \left\{t \geqslant T_{n}: Z(t)=\tau\right\}, n=1,2 \cdots,
\end{aligned}
$$

于是, 对于 $W$, 时刻 $T_{0}, T_{1}, T_{2} \cdots$ 构成了一列延迟更新时刻, 换句话说, $\left\{T_{0}, T_{1}, T_{2}, \cdots\right\}$ 形成一个延迟更新过程.

记在时间区间 $\left[0, T_{0}\right)$ 内的费用为 $C_{0, a}(\lambda, \tau)$, 又在 $\left[T_{n-1}, T_{n}\right)(n \geqslant 1)$ 内的费用记 为 $C_{n, \alpha}(\lambda, \tau)$, 则折扣因子为 $\alpha$ 的期望折扣总费用显然是

$$
C_{\alpha}(\lambda, \tau)=E_{(0,0)}\left(\sum_{n=0}^{\infty} C_{n, \alpha}(\lambda, \tau)\right)
$$

令

$$
\begin{aligned}
& T_{\tau}=\inf \{t \geqslant 0: W(t)=(\tau, M)\}, \\
& T_{\lambda}=\inf \{t \geqslant 0: W(t)=(\lambda, 0)\},
\end{aligned}
$$

由于 $\left\{T_{0}, T_{1}, T_{2}, \cdots\right\}$ 是一个延迟更新过程,因此对于 $n \geqslant 1$, 应用强 Markov 性得到

$$
\begin{aligned}
E_{(0,0)}\left(C_{n, \alpha}(\lambda, \tau)\right) & =E_{(0,0)}\left[\exp \left(-\alpha T_{n-1}\right) C_{1, \alpha}(\lambda, \tau)\right] \\
& =E_{(0,0)}\left(e^{-\alpha T_{\lambda}}\right)\left[E_{(\lambda, M)}\left(e^{-\alpha T_{\varepsilon}}\right) E_{(\tau, 0)}\left(e^{-\alpha T_{\lambda}}\right)\right]^{n-1} E_{(\lambda, M)}\left(C_{1, \alpha}(\lambda, \tau)\right] .
\end{aligned}
$$

现在, (2) 式变为

$$
C_{\alpha}(\lambda, \tau)=E_{(0,0)}\left(C_{0, \alpha}(\lambda, \tau)\right)+\frac{E_{(0,0)}\left(e^{-\alpha T_{\lambda}}\right) E_{(\lambda, M)}\left(C_{1, \alpha}(\lambda, \tau)\right)}{1-E_{(\lambda, M)}\left(e^{-\alpha T_{\tau}}\right) E_{(\tau, 0)}\left(e^{-\alpha T_{\lambda}}\right)} .
$$

显而易见, 我们只要能够算出 $E_{(0,0)}\left(C_{0 . \alpha}(\lambda, \tau)\right), E_{(0,0)}\left(e^{-\alpha T_{\lambda}}\right), E_{(\lambda, M)}\left(e^{-\alpha T_{\tau}}\right), E_{(\tau, 0)}\left(e^{\left.-\alpha T_{\lambda}\right)}\right.$ 和 $E_{(\lambda, M)}\left(C_{1, \alpha}(\lambda, \tau)\right)$ 的值来,就可以得到 $C_{\alpha}(\lambda, \tau)$ 的显式表示了.

由于注意到 $E_{(0,0)}\left(C_{0, \alpha}(\lambda, \tau)\right)$ 是和水库投入服务的时刻有关的, 因此为了方便起见，我 们不妨假定水库在时刻零即已投入服务,那么

$$
E_{(0,0)}\left(C_{0, \alpha}(\lambda, \tau)\right)=E_{(0,0)}\left[\int_{0}^{T_{\lambda}} e^{-\alpha t} g(Z(t)) d t\right] \text {. }
$$

再次应用强 Markov 性就得到

$$
\begin{gathered}
E_{(\lambda, M)}\left(C_{1, \alpha}(\lambda, \tau)\right)=K_{1} M+E_{(\lambda, M)}\left[\int_{0}^{T_{\tau}} e^{-\alpha t} g(Z(t)) d t\right]+K_{2} M E_{(\lambda, M)}\left(e^{-\alpha T_{\mathfrak{r}}}\right) \\
+E_{(\lambda, M)}\left(e^{\left.-\alpha T_{\mathfrak{r}}\right)} E_{(r, 0)}\left[\int_{0}^{T_{\lambda}} e^{-\alpha t} g(Z(t)) d t\right]-M E_{(\lambda, M)}\left[\int_{0}^{T_{\mathfrak{r}}} e^{-\alpha t} d t\right] .\right.
\end{gathered}
$$

现在令 $T-T_{2}$, 我们研究如下的费用泛函

$$
\begin{aligned}
R(x) & =E_{x}\left\{\int_{0}^{T} e^{-\alpha t} g(Z(t)) d t\right\} \\
& =E_{x}\left\{\int_{0}^{T} \exp \left(-\int_{0}^{t} a d s\right) g(Z(t)) d t\right\},
\end{aligned}
$$

其中 $0 \leqslant x \leqslant \lambda$. 根据熟知的结果， $R(x)$ 应为下述微分方程的解

$$
\frac{1}{2} \sigma^{2} R^{\prime \prime}(x)+\mu R^{\prime}(x)-\alpha R(x)+g(x)=0 \quad 0 \leqslant x \leqslant \lambda,
$$


其边界条件是 $R(\lambda)=0$ 和 $R^{\prime}(\lambda)=8$ （详细证明可参看 Karlin 和 Taylor ${ }^{[5]}$, p. 204 和 p. 385).

利用系数变易法, $R(x)$ 作为方程 (8) 满足上述边界条件的解, 是

$$
R(x)=\Phi_{\alpha}(\lambda, \mu, g(\cdot)) \delta_{\alpha}(x, \lambda, \mu)-\Phi_{\alpha}(x, \mu, g(\cdot)),
$$

这里 $\Phi_{\alpha}(x, \mu, g(\cdot))=\left\{\begin{array}{c}\gamma^{-1} \int_{0}^{x} g(t)\left\{\exp \left[(\gamma-\mu)(x-t) / \sigma^{2}\right]\right. \\ \left.-\exp \left[-(\gamma+\mu)(x-t) / \sigma^{2}\right]\right\} d t, \mu \neq 0, \\ 2\left(2 \alpha \sigma^{2}\right)^{-\frac{1}{2}} \int_{0}^{x} g(t) \operatorname{sh}\left[(2 \alpha)^{\frac{1}{2}}(x-t) / \sigma\right] d t, \mu=0,\end{array}\right.$

$\delta_{\alpha}(x, \lambda, \mu)=\left\{\begin{array}{l}\frac{(\gamma+\mu) \exp \left[(\gamma-\mu) x / \sigma^{2}\right]+(\gamma-\mu) \exp \left[-(\gamma+\mu) x / \sigma^{2}\right]}{(\gamma+\mu) \exp \left[(\gamma-\mu) \lambda / \sigma^{2}\right]+(\gamma-\mu) \exp \left[-(\gamma+\mu) \lambda / \sigma^{2}\right]}, \mu \neq 0, \\ \operatorname{ch~}\left[(2 \alpha)^{\frac{1}{2}} x / \sigma\right] / \operatorname{ch}\left[(2 \alpha)^{\frac{1}{2}} \lambda / \sigma\right], \quad \mu=0\end{array}\right.$

和

$$
\boldsymbol{r}=\left(\mu^{2}+2 \alpha \sigma^{2}\right)^{\frac{1}{2}} \text {. }
$$

特别地,

$$
\begin{aligned}
E_{(\tau, 0)} & {\left[\int_{0}^{T_{\lambda}} e^{-\alpha t} g(Z(t)) d t\right] } \\
& =\Phi_{\alpha}(\lambda, \mu, g(\cdot)) \delta_{\alpha}(\tau, \lambda, \mu)-\Phi_{\alpha}(\tau, \mu, g(\cdot)) .
\end{aligned}
$$

令 $1(\cdot)$ 为恒等于 1 的单位函数,则有

$$
\begin{aligned}
E_{(\tau, 0)} & \left(e^{-\alpha T} \lambda\right)=E_{(\tau, 0)}\left[1-\alpha \int_{0}^{T_{\lambda}} e^{-\alpha t} d t\right] \\
& -1-\alpha\left[\Phi_{\alpha}(\lambda, \mu, 1(\cdot)) \delta_{\alpha}(\tau, \lambda, \mu)-\Phi_{\alpha}(\tau, \mu, 1(\cdot))\right],
\end{aligned}
$$

如 $\tau=0$, 则由 (12) 和 (13) 式可分别得到 $E_{(0,0)}\left(C_{0, \alpha}(\lambda, \tau)\right)$ 和 $E_{(0,0)}\left(e^{\left.-\alpha T_{\lambda}\right)}\right.$ 的值.

同理可得

$$
\begin{aligned}
E_{(\lambda, M)} & {\left[\int_{0}^{T_{\tau}} e^{-\alpha t} g(Z(t)) d t\right] } \\
= & \Phi_{\alpha}(V-\tau, M-\mu, g(V-\cdot)) \delta_{\alpha}(V-\lambda, V-\tau, M-\mu) \\
& -\Phi_{\alpha}(V-\lambda, M-\mu, g(V-\cdot)) .
\end{aligned}
$$

再以单位函数 $1(\cdot)$ 代换 $(14)$ 式中的 $g(\cdot)$, 我们有

$$
\begin{aligned}
& E_{(\lambda, M)}\left(\int_{0}^{T_{\tau}} e^{-\alpha t} d t\right) \\
& =\Phi_{\alpha}(V-\tau, M-\mu, 1(\cdot)) \delta_{\alpha}(V-\lambda, V-\tau, M-\mu) \\
& \quad-\Phi_{\alpha}(V-\lambda, M-\mu, 1(\cdot)) . \\
& E_{(\lambda, M)}\left(e^{\left.-\alpha T_{\tau}\right)}\right. \\
& =1-\alpha\left[\Phi_{\alpha}(V-\tau, M-\mu, 1(\cdot)) \delta_{\alpha}(V-\lambda, V-\tau, M-\mu)\right. \\
& \left.\quad-\Phi_{\alpha}(V-\lambda, M-\mu, 1(\cdot))\right] .
\end{aligned}
$$

于是,将 (12)、(14)、(15) 和 (16) 等式代人 (6) 式就得到了 $E_{\langle\lambda, M)}\left(C_{1, \alpha}(\lambda, \tau)\right)$ 的值. 最后，以 (6)、(12)、(13) 和 (16) 等式代人 (4) 式, 就可以确定期望折扣总费用的显式表示 了. 特别地, 如果赔款率是形如 (1) 式的函数或者是一个多项式, 则泛函 $\Phi_{\alpha}$ 和 $C_{\alpha}(\lambda, \tau)$ 的 计算都是很简单的.

\section{\$3. 两种觉用的关系}

在作者前一篇论文中已经定义了长期平均费用 $C(\lambda, \tau)$ (见文献 $[1]$ 公式 (6)). 现在,我 
们要确定两种费用 $C_{\alpha}(\lambda, \tau)$ 和 $C(\lambda, \tau)$ 间的关系。

事实上, 容易证明

$$
\begin{aligned}
& \lim _{a \rightarrow+0} \Phi_{a}(x, \mu, g(\cdot)) \\
& -\left\{\begin{array}{l}
\mu^{-1} \int_{0}^{x} g(t)\left\{1-\exp \left[-2 \mu(x-t) / \sigma^{2}\right]\right\} d t, \quad \mu \neq 0, \\
2 \sigma^{-2} \int_{0}^{x} g(t)(x-t) d t, \quad \mu=0 .
\end{array}\right.
\end{aligned}
$$

这个极限正是文献 [1] 中由 (9) 式所确定的泛函 $\Phi(x, \mu, g(\cdot))$. 同时,

$$
\lim _{\alpha \rightarrow+0} \delta_{\alpha}(x, \lambda, \mu)=1,
$$

因而推出极限 $\lim _{a \rightarrow+0} R(x)$ 存在并且等于文献 [1] 中的 $W(x)$.

最后, 可以直接地证明

$$
\lim _{\alpha \rightarrow+0} \alpha C_{\alpha}(\lambda, \tau)-C(\lambda, \tau) .
$$

(17) 式实际上是文献 [3] 中结果的推广.

\section{参文献}

[1] Lam Yeh, Optimal control of a finite dam-Average cost case., J. Appl. Prob., 22(1985).

[ 2 ] Faddy, M. J., Optimal control of finite dams: discrete (2-stage) output procedure, J. Appl. Prob., 11 (1974), 111-121.

[ 3 ] Zuckerman, D., Two-stage output procedure of a finite dam., J. Appl. Prob., 14(1977), 421-425.

[ 4 ] Attia, F. A. and Brockwell, P. J., The control of a finite dam., J. Appl. Prob., 19(1982), 815-825.

[5] Karlin, S. and Taylor, H. M., A Second Course in Stochastic Processes, Academic Press, New York, 1981. 